\title{
La Entomología Forense en España: pasado, presente y perspectivas de futuro.
}

\author{
Forensic Entomology in Spain: past, present and future \\ perspectives.
}

\author{
A. Gómez-Gómez¹, D. Martín-Vega1', C. Botías-Talamantes', \\ A. Baz-Ramos² y LM. Díaz-Aranda²
}

\section{RESUMEN}

La entomología forense -estudio de los insectos que aparecen en los cadáveres- es una disciplina relativamente joven en cuanto a su aplicación. En España ha evolucionado lentamente, experimentando un notable crecimiento en las últimas décadas, tanto en el número de trabajos como en el de grupos de investigación dedicados a ello, adquiriendo un estatus cada vez más importante como disciplina forense.

En este trabajo se presentan los resultados de una revisión bibliográfica en la que se ha analizado con detalle la evolución de las publicaciones relacionadas con la entomología forense en España. Se incluye además un anexo con las referencias de todos los artículos recopilados, clasificados según sus características (divulgación científica, educación o formación, aplicación en casos reales e investigación en entomología forense).

Finalmente se analiza el estado actual de esta disciplina en nuestro país, considerando la faceta educativa, así como los principales lugares donde se investiga y aquellos donde se aplica la entomología forense en la resolución de casos reales.

Palabras clave: Aplicación en casos reales, bibliografía, divulgación, entomología forense, evolución histórica, formación, investigación.

Cuad Med Forense 2007; 13(47):21-32

Este trabajo ha sido financiado por el Ministerio de Ciencia y Tecnología (Proyecto de investigación BOS2003-00400) y por la Universidad de Alcalá (Proyecto de investigación PI2003/016).

Fecha de recepción: 9.MAR.07

Fecha de aceptación: 4.MAY.07

Correspondencia: Aída Gómez-Gómez. Dpto. de Zoología y Antropología Física. Facultad de Biología. Universidad de Alcalá. Ctra. Barcelona, km. 33,6. 28871 Alcalá de Henares (Madrid). Teléfono: 9188549 22. Fax: 918855080. E-mail: aida.gomez@uah.es.

1 Licenciado en Biología. Personal Investigador en Formación. Universidad de Alcalá. Alcalá de Henares (Madrid).

2 Doctor en Biología. Profesor Titular. Universidad de Alcalá. Alcalá de Henares (Madrid). 


\section{INTRODUCCIÓN:}

La entomología forense es la disciplina encargada del estudio de los artrópodos que se encuentran en los cadáveres con el propósito de proporcionar información útil en las investigaciones policiales y/o judiciales, siendo la aportación más importante la estimación del intervalo postmortem.

Aunque se trata de una disciplina forense con casi dos siglos de uso, sigue siendo una disciplina joven, ya que su evolución ha sido lenta y su desarrollo ha dependido de la dedicación de un número de científicos relativamente bajo en comparación con otros campos médicos y paramédi$\cos [\mathrm{I}]$. En la actualidad la entomología forense está ganando cada vez más reconocimiento internacional [2], y son muchos los retos planteados para el futuro, siendo uno de los más importantes la combinación de los resultados obtenidos en las investigaciones con el trabajo en casos reales [3].

Aunque el primer episodio que documenta la relación entre insectos y cadáveres se remonta al siglo XIII [4], no es hasta siglos después cuando se comienza a estudiar la relación entre los insectos y los cadáveres. Francesco Redi en Italia [5] o más tarde el español Mateo Orfila en compañía del francés Octave Lesueur en Francia $[6,7]$ estudiaron la presencia de determinados dípteros en los cadáveres, pero no contemplaron una aplicación práctica para la estima de la data de muerte.

En España, son varios los autores que en esa época dejan constancia de las observaciones de insectos necrófagos sobre cadáveres humanos $[8,9,10]$, pero sin entrar en estudios detallados ni en su posible aplicación médico-legal.

Es Bergeret quien aplica por vez primera la entomología para establecer la data de la muerte en un caso de infanticidio [I I], suceso que fue recogido por Mata [I 2]. Años después, Graells fue el primer español en profundizar en el campo desde un punto de vista aplicado, haciéndose eco de los trabajos realizados por Mégnin [13], quien describió por primera vez la sucesión que establecen los insectos en los cadáveres a medida que se produce el proceso de descomposición, resaltando la utilidad de la entomología en las prácticas médico legales.

Muchos otros autores trataron la presencia de los insectos en los cadáveres y la utilidad de la entomología en los casos forenses, aunque pocos realizaron trabajos de investigación dentro de esta disciplina. Además, la mayoría de estas publicaciones se limitaron a exponer los resultados de Mégnin [14]; de hecho, algunas publicaciones formativas o de divulgación recientes han continuado reproduciendo los resultados del veterinario francés [15]. Por el contrario, otros autores españoles cuestionaron la validez de los resultados de Mégnin, resaltando la importancia de conocer la fauna cadavérica en las diferentes regiones geográficas; ejemplo de ellos fueron Lecha-Marzo [16], Álvarez Herrera [17], o Piga, que escribió: "Bastantes de las aseveraciones hechas por Mégnin, tomadas como artículo de fe y copiadas por todos los autores sin procurar comprobar su exactitud, van siendo en estos últimos años rectificadas, cosa que no tiene nada de extraño, puesto que aun siendo completamente exacto todo lo observado por el sabio entomólogo, es lógico deducir la diferencia y variabilidad de la fauna cadavérica en diferentes regiones" [18].

A pesar de conocerse la necesidad de realizar estudios biogeográficos, los trabajos de investigación llevados a cabo en España han sido muy escasos y puntuales [1 9, 20, 21 , 22], y muchas veces centrados únicamente en observaciones personales [16, 23, 24, 25].

En la actualidad son varios los grupos españoles que empiezan a realizar trabajos de investigación sobre la fauna de interés forense y, aunque ya se han aportado datos sobre sucesión a nivel regional [22, 26], todavía quedan lagunas en muchos aspectos, tanto en lo referente a las zonas geográficas, como en las distintas áreas de esta disciplina. 
Este trabajo pretende dar una visión de la evolución de la entomología forense en España a lo largo de la historia, así como de su situación actual en distintos aspectos, tales como la investigación, su aplicación a casos reales y, por último, el educativo o de formación. Se incluye además un listado bibliográfico que recopila los trabajos de autores españoles sobre esta disciplina.

\section{MATERIAL Y MÉTODOS:}

- REVISIÓN BIBLIOGRÁFICA.

Se ha llevado a cabo una búsqueda bibliográica recopilando todos los artículos publicados sobre las investigaciones realizadas en España, consultando para ello diversas bases de datos a través de la red como Biological Abstracts, CINDOC, ISI Web of Knowledge, JSTOR, Medline, PubMed, ScienceDirect o SpringerLink.

Se han consultado también todos los libros y revistas de medicina legal y otras disciplinas científicas a los que se tuvo acceso en diferentes bibliotecas universitarias de la Comunidad de Madrid, realizando un vaciado sistemático en busca de referencias a la entomología forense.

Una vez recopilado todo el material, las publicaciones se han clasificado de la siguiente manera:

- por décadas

- por tipo de material consultado (libros o revistas)

- por categorías, según el siguiente criterio subjetivo:

a. Divulgación: artículos de divulgación científica.

b. Educación o formación, entendiéndose como tal aquellos artículos que describen los principios en los que se basa la entomología forense.

c. Aplicación: descripción de casos reales en los que se ha aplicado la entomología.

d. Investigación: artículos científicos donde se publican los resultados de las investigaciones realizadas en distintas áreas de la entomología forense y cuyo objetivo es la posible aplicación de esos resultados a la resolución de casos reales.

En el último grupo no se han incluido aquellos trabajos de investigación básica en entomología, tales como estudios de ecología, biología, taxonomía o faunística de los diferentes grupos de insectos necrófilos, que pudieran resultar de gran utilidad en las investigaciones forenses [2 I, 27, 28, 29]. Tampoco se han incluido las numerosas conferencias y contribuciones a congresos por la dificultad de cuantificarlas.

\section{- La entomología forense en la ACTUALIDAD.}

Con el propósito de analizar la situación de la entomología forense en la actualidad en nuestro país, se han localizado todos aquellos lugares en los que se investiga y/o se aplica esta disciplina.

Por otro lado se ha llevado a cabo una búsqueda de aquellos centros (universidades, institutos de criminología y de medicina legal, etc.) en los que se imparte formación en distintas áreas forenses, con el objetivo de comprobar en cuáles de ellos se contempla la enseñanza de la entomología forense.

\section{RESULTADOS:}

- REVISIÓN BIBLIOGRÁFICA.

Se han recopilado 104 publicaciones, cuyas referencias se muestran en el Anexo, clasificadas según el criterio explicado en el apartado de material y métodos. 
En cuanto a la evolución histórica, analizar el número de artículos científicos publicados es una buena manera de estimar cómo ha progresado una disciplina a lo largo de la historia.

Como puede observarse en la Gráfica I, el número de publicaciones sobre entomología forense en España fue escaso desde la aparición de esta disciplina en la segunda mitad del siglo XIX. Es a partir de las dos últimas décadas del siglo $X X$ cuando el número de trabajos experimentales, y con ello el número de publicaciones, se ha incrementado considerablemente, una tendencia que continúa en la actualidad y que coincide con el auge de esta disciplina en otros países europeos y americanos.

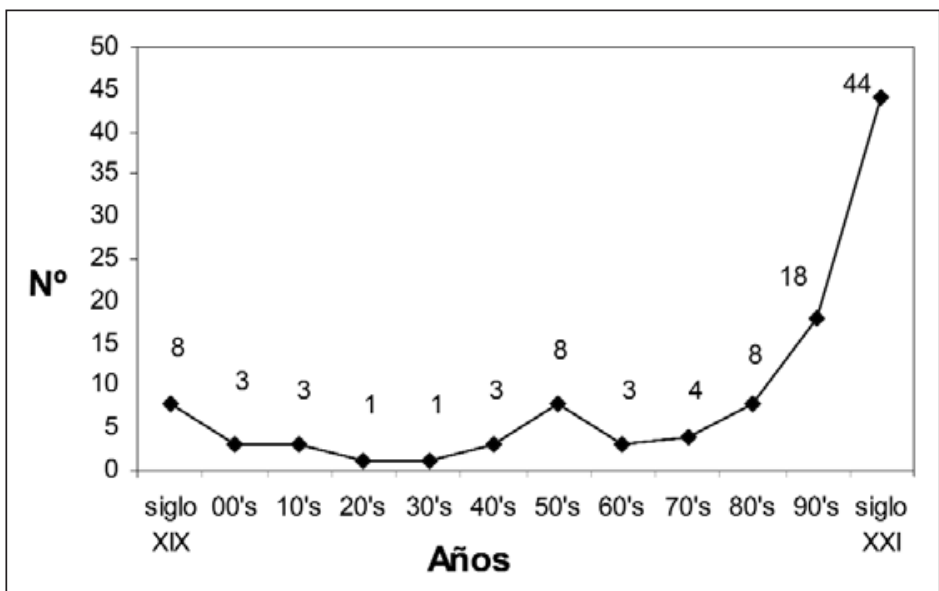

Gráfica 1. Número de publicaciones sobre Entomología Forense en España a lo largo de la historia.

En cuanto a las fuentes consultadas, el Diagrama de Sectores I.I. hace referencia a los libros de medicina legal revisados. De un total de 96 libros, sólo se encontraron referencias sobre entomología forense en 33 de ellos (aprox. 34\%), siendo la mayoría de la segunda mitad del siglo $X X$. Algunos de estos libros sólo dedican a la entomología forense unas pocas líneas, pero en el resto pueden encontrarse capítulos relativamente extensos en relación a esta disciplina.

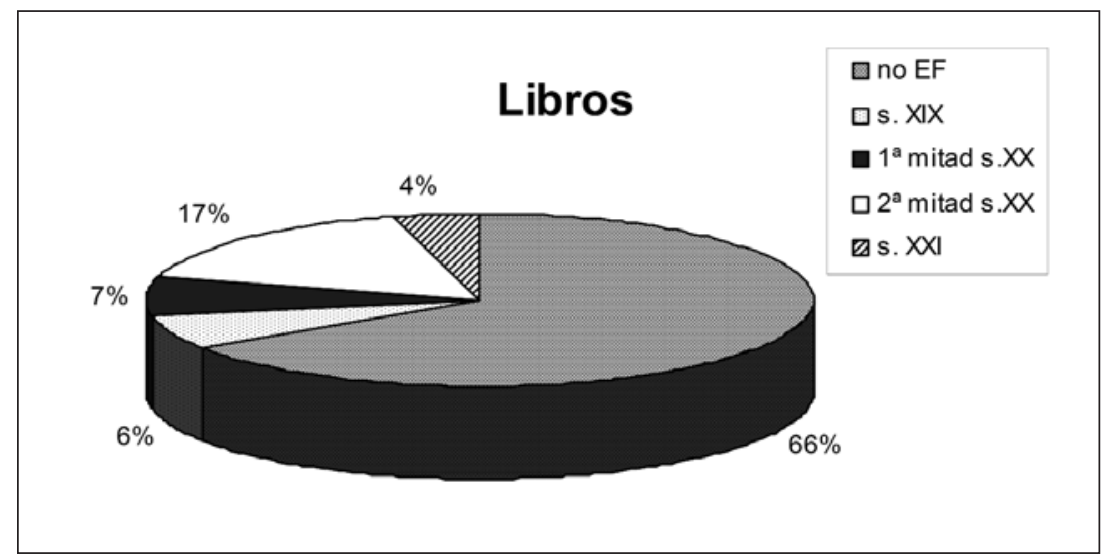

Diagrama de Sectores 1.1. Libros de medicina legal consultados. Aquellos que contienen referencias sobre Entomología Forense (34\%) han sido clasificados según su fecha de publicación. 
En el caso de las revistas científicas (Diagrama de Sectores 1.2.), el porcentaje de referencias es mucho más pequeño (aprox. 13\%). Se consultaron 648 números de 65 revistas científicas diferentes, encontrando referencias únicamente en 70 de ellos, la mayoría publicados en la segunda mitad del siglo XX.

En lo referente al tipo de publicación y según las cuatro categorías descritas en el apartado de material y métodos, puede observarse que la mayoría de ellas (43\%) corresponden a publicaciones de carácter educativo, el $36 \%$ corresponde a trabajos de investigación, un I4\% son publicaciones de carácter divulgativo y sólo el 7\% describen casos reales en los que se aplicó la entomología.

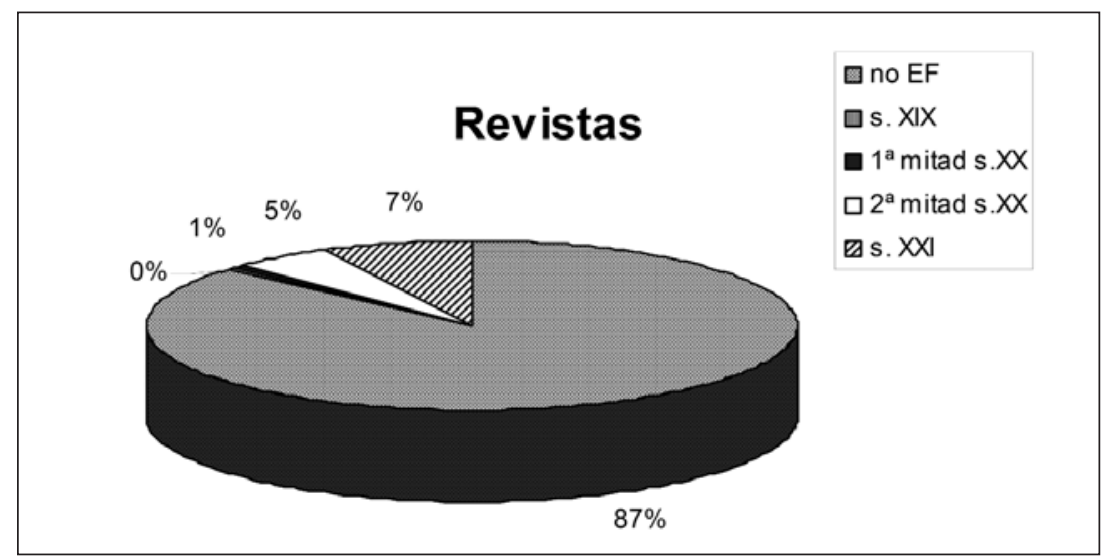

Diagrama de Sectores 1.2. Revistas científicas consultadas. Aquellos volúmenes que contienen referencias sobre Entomología Forense (13\%) han sido clasificados según su fecha de publicación.

\section{- La entomología Forense en la actualidad.}

En cuanto a la Investigación, en la actualidad son cinco las universidades de todo el país en las que se investiga en el campo de la entomología forense: Universidad de Alcalá, Universidad de Alicante, Universidad de Murcia, Universidad del País Vasco y Universidad de Cádiz. Los proyectos de investigación llevados a cabo en las cuatro primeras se han centrado, hasta el momento, en los campos más básicos de esta disciplina forense, como son la catalogación de la fauna de interés forense de determinadas zonas geográficas, el establecimiento de las sucesiones de insectos a medida que avanza el proceso de descomposición y el estudio del ciclo de desarrollo de las especies de dípteros que aparecen con más frecuencia en casos forenses.

Por otro lado, en el caso de la Universidad de Cádiz, se trabaja en la identificación de especies forenses a través de métodos moleculares, ya que las técnicas de ADN contribuyen de una manera rápida e inequívoca a la identificación de las especies implicadas en los casos.

Aplicación. Existen varios centros en España que aplican la entomología forense en la resolución de casos reales, entre ellos cabe destacar el Instituto Anatómico Forense [30]; el Laboratorio de Antropología Forense de la Comisaría General de Policía Cientíica; varios departamentos del Instituto Nacional de Toxicología y Ciencias Forenses y el Laboratorio Forense para la Vida Salvaje, en el que se aplica la entomología forense para resolver casos relacionados con especies animales protegidas. Estos centros reciben muestras procedentes de distintos puntos del país.

Además, investigadores de las Universidades de Alicante, Murcia y País Vasco han colaborado o colaboran con alguno de estos centros en la resolución de casos reales y en la elaboración de informes periciales. 
Formación. En cuanto a la actividad educativa, se han encontrado 58 cursos relacionados con criminología, criminalística y ciencias forenses en general en cerca de 40 centros diferentes. Sólo 16 de estos cursos incluyen la entomología forense en sus programas, y únicamente 4 profundizan realmente en esta disciplina, sin obtenerse en ningún caso un título oficial de entomólogo forense. No se han contabilizado aquellos cursos que no se imparten en la actualidad, así como seminarios o conferencias puntuales, que como ya se comentó anteriormente, resultan imposibles de cuantificar.

\section{DISCUSIÓN Y CONCLUSIONES:}

- REVISIÓN BIBLIOGRÁFICA.

En España los trabajos experimentales sobre entomología forense han sido muy escasos hasta hace una década aproximadamente, si bien no se ha ignorado la importancia de esta disciplina en vista de la cantidad de referencias de carácter formativo encontrada en libros y revistas de medicina legal.

Esta situación es paralela a la evolución de la disciplina en todo el mundo, ya que aunque la utilización de los insectos como herramienta forense comenzó en Europa de forma metódica a finales del siglo XIX [1 1, 14], no ha sido hasta hace un par de décadas cuando ha logrado su reconocimiento como disciplina de utilidad en las investigaciones policiales, de hecho Vincent et al. [3। ] recopilan toda la bibliografía publicada sobre el tema hasta 1983, presentando únicamente 329 referencias. Desde dicha revisión, las distintas áreas de la Entomología Forense han progresado de forma considerable y con ello el número de artículos científicos publicados [4]. Con todo esto, y a pesar del interés surgido en los últimos años en España, el conocimiento en otros países es muy superior [32].

Respecto a la descripción de casos resueltos con ayuda de la entomología, los artículos publicados no reflejan el número real de casos en los que se ha aplicado esta disciplina, al menos en algunos de los centros en los que la entomología forense es una herramienta habitual en la estimación del intervalo postmortem (Magaña, com. pers.).

\section{- La entomología forense en la Actualidad.}

En los últimos años ha aumentado el número de lugares y grupos de investigación, algunos de ellos dotados con financiación económica procedente de la administración. Sin embargo, todavía falta mucha información, tanto de los temas a tratar, como de las zonas geográficas a cubrir, lo cual obliga a recurrir a datos de otros países para la resolución de casos, con las implicaciones negativas que ello conlleva.

Además de las tres líneas básicas en las que se centran los proyectos de investigación llevados a cabo en nuestro país (faunística, sucesiones y desarrollo de dípteros), existen otras líneas necesarias en las investigaciones policiales y/o judiciales, que se desarrollan en otros países, tales como toxicología, $\mathrm{ADN}$, comportamiento, efecto de diversas variables, etc, de las que todavía no se han realizado estudios en profundidad en España.

A su vez, es necesario promover trabajos de ecología, biología, faunística y taxonomía de los grupos de insectos de interés forense de nuestra fauna, puesto que su ayuda es inestimable para la adecuada aplicación de esta disciplina.

Respecto a la formación en entomología forense, se ha comprobado que resulta insuficiente, ya que son escasos los programas de estudio que la incluyen en su temario, y los que sí lo hacen profundizan poco, no existiendo un certificado oficial de entomólogo forense como ya se ha mencionado anteriormente. En este sentido, cabe destacar la reciente creación del Instituto Universitario de Ciencias Policiales, fruto de un convenio entre el Ministerio del Interior y la Universidad de Alcalá, 
entre cuyos objetivos se encuentra ampliar la formación de los cuerpos de seguridad del Estado en distintas disciplinas forenses.

En los últimos años ha surgido cierto interés popular por la entomología forense, debido en parte a la aparición de series televisivas, lo cual tiene el aspecto positivo de dar a conocer la importancia y utilidad de ésta y otras disciplinas en las investigaciones policiales. Sin embargo, esto ha generado la aparición de bastante intrusismo, con los problemas que genera la publicación de datos de dudosa fiabilidad.

Aunque la entomología forense ha evolucionado considerablemente en muchos aspectos en España en las dos últimas décadas, hay todavía un largo camino por recorrer, especialmente en cuanto a investigación en las diversas áreas de esta disciplina, en diferentes zonas geográficas.

\section{AGRADECIMIENTOS:}

Agradecemos a todas las personas que nos han atendido y facilitado información necesaria para la elaboración de este trabajo, especialmente a la Dra. Concepción Magaña del Instituto Anatómico Forense, a los Dres. Ana Isabel Martínez-Sánchez y Santos Rojo de la Universidad de Alicante, a la Dra. Ma Dolores García de la Universidad de Murcia, y al Dr. Manuel Castillo por la inestimable ayuda prestada y la amabilidad con que nos atendieron.

También queremos mencionar al personal de las distintas bibliotecas a las que hemos acudido por facilitarnos el trabajo.

\section{ANEXO:}

Divulgación

- Alcaraz, R.: Datación del cadáver antiguo. Sector Seguridad. 2002; 8: 28-32

- Alcaraz, R.: Putrefacción cadavérica. Sector Seguridad. 2002; 6:

$36-40$.

- Antón Barberá, F.: La entomología forense en la investigación policial. Ciencia Policial. 2000; 54: 21-29.

- Antón Barberá, F.: Nuevos confidentes de la policía: los insectos.

Revista Agente de Policía. 2003; 4 (17): 51-56.

- Aznar Cervantes, S. D.: Phoridae: Todo un reto para la entomología forense. Eubacteria. 2005; 17: 19-21.

- Berg, E. van den: Se ha escrito un crimen. National Geographic España. 2001; 8 (5): 64-74.

- Castillo, M.: El papel de las moscardas en la entomología forense: dípteros necrófagos y colonización de carroñas. Quercus. 2001; 186: 24-27.

- Castillo, M.: Entomofauna carroñera: la magia de convertir lo muerto en vivo. Ibón. 2001; 13: 22-28.

- Coperías, E. M.: Los insectos ayudan a la policía. La mosca delató al asesino. Muy interesante. 1996; 184: 67-69.

- Coperías, E. M.: La pista del crimen. Así ayudan los insectos a la polićá. Muy interesante. 2004; 277: 48-56.

- González Peña, L.: En la vanguardia de las ciencias forenses. Revista Polićía. 2002; 161: 40-48.

- Magaña, C.: Entomología forense. Insectarium virtual (revista electrónica). 2003.

- Moreno Martínez; Piera Pellicer, J. A.; Antón Barberá, F.; Mené Moreno, C.; Mulet Falcó, E.; Tirado Darder, J. J.: La entomología aplicada a la investigación forense. Enfermería Integral. 2000; 52: 7-II.

- Romera, E.; Arnaldos, M. I.; García, M. D.: Entomología forense... esa gran desconocida. Eubacteria. 2002; 10: 8-9.

- Viejo Montesinos, J. L.; Romero López, P. S.: Entomología forense. Quercus. 1992; 82: 33-35.

\section{Educación o Formación}

- Álvarez Herrera, A.: Auxiliares de la putrefacción. Salvat. Barcelona, 1940. 28-30.

- Anónimo.: Médicos forenses [Temario para oposiciones]. Volumen I. [s. n.]. [s. I.], 1935. 772-778.

- Antón Barberá, F.; de Luis y Turégano, J. V.: Policía científica. $3^{\mathrm{a}}$ ed. Tirant lo Blanch. Valencia, 1998. 917-918.

- Arnaldos, M. I.; Romera, E. García, M. D.; Luna, A.: Protocolo para la recogida, conservación y remisión de muestras entomológicas en casos forenses. Cuadernos de Medicina Forense. 200I; 25: 65-73.

- Aso Escario, J.; Corrons Perramon, J.; Cobo Plana, J. A.: El intervalo postmortal. Interés médico, policial, legal y forense. Masson. Barcelona, 1998. 51-55.

- Ayala y Galán, P. de.: Fauna cadavérica según Megnin (material gráfico). Instituto de Medicina Legal, Toxicología y Psiquiatría. Madrid, 1918.

- Barahona Holgado, I.: Lecciones de medicina legal. Establecimiento Tipográfico de Marcelino Rodríguez. Salamanca, 1908. 426-433.

- Calatayud Carral, F.: Evolución de los fenómenos de putrefacción de la cámara pulpar en relación con la cronotanatología. Anales Españoles de Odontoestomatología. 1944; 3 (II): 945-952. 
- Capó, M. A.; Peinado, M. V.; Mateos, J.; Anadón Baselga, M. J.: Entomofauna cadavérica establecida al aire libre. Medicina Balear. 2004; 19 (2): 29-38.

- Casas Sánchez, J. D.; Rodríguez Albarrán, M.S.: Manual de medicina legal y forense. Colex. Madrid, 2000. 1200-1202.

- Chavarría Puyol, H. M.: Bioética y ciencias forenses en Internet. Entomología forense. Cuadernos de Medicina Forense. 1996; 6: 56-57.

- Fernández Cabeza, J.; Gisbert Calabuig, J. A.; Domínguez Martínez, J.; Pérez de Petinto y Alonso Martínez, J. M.: Autopsia Médico-Forense Negativa. Anales de Medicina Forense de la Asociación Española de Médicos Forenses. 1962. 49-129.

- Fernández Cuesta y Porta, N.: Autopsia judicial: Compendio médicolegal necróptico para uso de magistrados, jueces, médicos forenses, titulares de sanidad militar y de sanidad de la armada, y en general, para cuantas personas tengan que intervenir más o menos directamente en una diligencia de autopsia. Romero Editor. Madrid, 1895. 146.

- Galante, E.; Marcos-García, M. A.: Detritívoros, Coprófagos y Necrófagos. Boletín de la Sociedad Entomológica Aragonesa. 1997; 20: 57-64.

- Galante, E.; Marcos-García, M. A. Decomposer insects. En: Encyclopedia of Entomology. J. L. Capinera, ed. Kluwer Academic Publisher. Dordrecht, 2004. 664-674.

- Giner Alberola, S.: Estudio de la putrefacción. Entomología cadavérica. En: Antropología criminológica. Fernando Rodes Lloret y Juan Bautista Martí Lloret, eds. Universidad Miguel Hernández. Elche, 2001. 91-109.

- Gisbert Calabuig, J. A.: Medicina legal y práctica forense. Revista de Medicina Legal. 1958; I3 (144-145): 137-216.

- Gisbert Calabuig, J. A.: Medicina Legal y Toxicología. Fundación García Muñoz. Valencia, 1977. 270-272.

- Goff, M.L. García, M. D.; Arnaldos M. I.; Romera, E. \& Luna,A. 2004. "Entomología cadavérica: Fundamentos y aplicación. Referencia a la entomología española". En: Gisbert Calabuig. Tratado de Medicina Legal y Toxicología, $6^{a}$ edición, pp. 253-273. Ed Masson, Barcelona.

- González Peña, C. F.: Los insectos y la muerte. Boletín de la Sociedad Entomológica Aragonesa. 1997; 20: 285-290.

- Graells, M.: Entomología judicial. Revista de los Progresos de las Ciencias Exactas, Físicas y Naturales. Madrid. 1886; 2I: 458-47I.

- Hinojal Fonseca, R.; Serrano Hernández, J. M.: Actuación del médico en intervenciones médico-legales de urgencia. Jarpyo. Madrid, 1995. 15.

- Lecha Martínez, L.: Manual de medicina legal. Imprenta y Librería de Nicolás Moya. Madrid, 1912. 228-241.

- Lecha-Marzo, A.: Tratado de autopsias y embalsamamientos. Editorial Los Progresos de la Clínica. Madrid, 1917. 79-90.

- López Gómez, L.; Gisbert Calabuig, J. A.: Tratado de medicina legal. Saber. Valencia, 1967. 430-435.

- López Sañudo, L.: Estudios postmortem verificados en el necroscomio y cementerio de Torrero en Zaragoza [Manuscrito]. (Tesis doctoral). Universidad Central. Madrid, 1891. 26-28.
- Magaña, C.: La entomología forense y su aplicación a la medicina legal. Data de la muerte. Boletín de la Sociedad Entomológica Aragonesa. 2001; 28: 49-57.

- Martínez-Calcerrada, L.: Derecho médico. Volumen I. Tecnos. Madrid, 1986. 710-713.

- Mata, P.: Vade Mecum de Medicina y Cirugía Legal. Volumen II. Imprenta de Manini y compañía. Madrid, 1844. 66 / 72.

- Mata, P.: Tratado de Medicina y Cirugía Legal teórica y práctica seguida de un compendio de Toxicología. Tomo II. Cuarta edición. Bailly-Bailliere. Madrid, 1866. 924-1012.

- Medina y Ramos, M.: Noticia sobre entomología judicial. Anales de la Sociedad Española de Historia Natural (Actas). 1888; 17: 100-10I.

- Molina Caballero, J.: Proceso bacteriano de la putrefacción cadavérica. Revista de Medicina Legal. 1953; 8 (90-91): 497-504.

- Muñoz Tuero, L. M.: Apuntes de medicina legal. Tanatología I. Departamento de Medicina Legal. Madrid, 1975. 109-II3.

- Nadal Moncadas, B.: Métodos de conservación cadavérica y sus aspectos legales y sanitarios (Tesis doctoral). Universidad Complutense de Madrid. Madrid, 1985. 38-40.

- Nájera Angulo, L.: La lucha contra las moscas (monografía para médicos). [s. n.]. Madrid, 1947. 5-23 / 24-60.

- Osuna Carrillo de Albornoz, E. J.: Nuevas perspectivas en la recogida e investigación de los indicios hallados en el acto de inspección ocular y levantamiento del cadáver. En: Seminario sobre la diligencia de inspección ocular, identificación y levantamiento del cadáver. Ministerio de Justicia. Madrid, 1991. 51-71.

- Piga, A.: Medicina Legal de Urgencia. La autopsia judicial. Editorial Mercurio. Madrid, 1928. 169-179.

- Reverte Coma, J. M.: Antropología Forense. Ministerio de Justicia, Secretaría General Técnica, Centro de Publicaciones. Madrid, 1991. 399-453.

- Rodríguez Albarrán, M. S.: Entomología cadavérica. En: Lecciones de medicina legal. Cronotanatodiagnóstico. Fernando Bandrés, ed. Dpto. Toxicología y Legislación Sanitaria, Facultad de Medicina (UCM). Madrid, 2001. 69.

- Royo-Villanova, R.; Aznar, B.; Piga, B.: Lecciones de medicina legal II. Marbón. Madrid, 1952.33-39.

- Sánchez Sánchez, J. A.; Villalaín, J. D.: Evolution et etat actuel de l'entomologie medico-legale en Espagne. XIVth Congress of the International Academy of Legal Medicine and Social Medicine. Liège, Bélgica. 1988; 303-307.

- Solís Claras, L.: Estudio acerca de la conservación provisional de los cadáveres y piezas anatómicas [Manuscrito]. (Tesis doctoral). Universidad Central. Madrid, 1885. 26-28 / II7.

- Villalaín Blanco, J. D.: Policía científica. El autor. Madrid, 1981. II7-II9.

- Villalaín Blanco, J. D.; Ramos Almazán, M. T.: Consideraciones médico-legales en relación al cuerpo momificado de Colmenar Viejo. Revista Española de Medicina Legal. 1981; 26-27: 69-80.

- Villanueva, E.: Cronotanatodiagnóstico. Métodos, crítica. En: Estudios dedicados a Juan Peset Aleixandre, Tomo III. Universitat de Valencia. Valencia, 1982. 779-809. 


\section{Investigación}

- Agudo Ordóñez, J.; Amestoy Jurado, M. P.; Sancho Ruíz, M.: La influencia de las condiciones ambientales sobre el desarrollo de la fauna cadavérica. Estudio de dos casos. Cuadernos de Medicina Forense. 1998, 14:49-55.

- Arnaldos, I.; Romera, E.; García, M. D.; Luna, A.: An initial study on Sarcosaprophagous Diptera (Insecta) succession on carrion in southern Iberian Peninsula. International Journal of Legal Medicine. 2001; II4: 156-162.

- Arnaldos, M. I.; Romera, E.; García, M. D.; Baquero, E.: Nuevos datos sobre la fauna de Mymaridae (Hymenoptera, Chalcidoidea) de la Península Ibérica de una comunidad sarcosaprófaga. Boletín de la Asociación española de Entomología. 2003; 27 (I-4): 225-228.

- Arnaldos M. I.; Romera, E.; Presa, J. J.; Luna, A \& García, M. D.: Studies on seasonal arthropod succession on carrion in southeastern Iberian Peninsula. International Journal of Legal Medicine. 2004; II8: 197-205

- Báguena, L.: Algunas notas sobre entomología médico-legal. Graellsia. 1952; 10: 67-101.

- Bahillo de la Puebla, P.; Saloña Bordas, M. I.; López Colón, J. I.: Confirmación de la presencia de Omosita depressa (Linnaeus, 1758) en la Península Ibérica (Coleoptera: Nitidulidae). Boletín de la Sociedad Entomológica Aragonesa. 2004; 34: I61-162.

- Berzosa, J.; Arnaldos, M. I.; Romera, E.; García, M. D.: Tisanópteros (Insecta: Thysanoptera) de una comunidad sarcosaprófaga en el sureste español. Boletín de la Real Sociedad Española de Historia Natural (Sección Biológica). 2001 ; 93 (3-4): 183-194.

- Castillo, M.: Artrópodos presentes en carroña de cerdos en la comarca de la Litera (Huesca). Boletín de la Sociedad Entomológica Aragonesa. 2001; 28: 133-140.

- Castillo, M.: Determinación de los estados y la velocidad de descomposición en cadáveres de cerdos y su aplicación a la entomología forense. Cuadernos CEHIM0. 2001; 29: 96-106.

- Castillo, M.: Principales especies de coleópteros necrófagos presentes en carroña de cerdos en la comarca de la Litera (Huesca). Graellsia. 200I; 57 (I): 85-90.

- Castillo, M.: Principales especies del orden Hymenoptera presentes en carroña de cerdos en la comarca de La Litera (Huesca). Zapateri: Revista Aragonesa de Entomología. 200I; 9: 89-92.

- Castillo, M.: Estudio de la entomofauna asociada a cadáveres en el Alto Aragón (España). Monografías de la Sociedad Entomológica Aragonesa. Zaragoza, 2002; 6: I-93.

- Domínguez Martínez, J.; Gómez Fernández, L.: Observaciones en torno a la entomología tanatológica. Aportación experimental al estudio de la fauna cadavérica. Revista Ibérica de Parasitología. 1957; 17 (I-2): 3-30.

- Domínguez Martínez, J.; Gómez Fernández, L.: Momificación cadavérica y particularmente rápida, operada bajo la acción de numerosas larvas de Chrysomyia albiceps Wiedemann, 1819. Revista Ibérica de Parasitología. 1963; 23: 43-62.
- García-Arribas, 0.; Pérez-Calvo, M.; Ribas-Ozonas, B.; Labrousse, H.: Diptera larvae biotest as alternative assy for detection of toxicity. Proceedings of the First European Forensic Entomology Seminar. 2002. 100-II2.

- García-Rojo, A. M.: Estudio de la sucesión de insectos en cadáveres en Alcalá de Henares (Comunidad Autónoma de Madrid) utilizando cerdos domésticos como modelos animales. Boletín de la Sociedad Entomológica Aragonesa. 2004. 34: 263-269.

- Gómez Fernández, L.: Los primeros estados de las larvas de las miasis humanas en el grupo Calliphora. (Diptera Cyclorrapha). Revista Ibérica de Parasitología. 1953; 13 (3): 259-288.

- Gómez Fernández, L.: Los primeros estados de las larvas de las miasis humanas en el grupo Phaenitia (Lucilia). Diptera Cyclorrapha. Revista Ibérica de Parasitología. 1958; 18 (I): 19-49.

- Martínez, M. D.; Arnaldos, M. I.; García, M. D.: Datos sobre la fauna de hormigas asociadas a cadáveres (Hymenoptera: Formicidae). Boletín de la Asociación Española de Entomología. 1997; 21 (3-4): 28I-283.

- Martínez, M. D.; Arnaldos, M. I.; Romera, E.; García, M. D.: Los Formicidae de la comunidad sarcosaprófaga en el Mediterráneo Occidental. Anales de Biología. 2002; 24: 33-44.

- Orfila, M. J. B.: Tratado de Medicina Legal, traducido de la cuarta edición y arreglado a la legislación española por el doctor Don Enrique Ataide. Tomo I. Imprenta de Don José María Alonso. Madrid, 1847. 444- 728.

- Pérez de Petinto y Bertomeu, M.: La miasis cadavérica en la esqueletización. Volumen Extraordinario del Primer Centenario (187I-197I) de la Real Sociedad Española de Historia Natural. 1975; Tomo II (Trabajos Científicos de Biología): 463-482.

- Pérez-Romero, S.; Marcos-García, M. A.; Rojo, S.: Comparative morphology of early stages of two Mediterranean Sarcophaga Meigen, 1826 (Diptera; Sarcophagidae) and a review of the feeding habits of Paleartic species. Micron. 2006; 37: 167-179.

- Prieto, J. L.; Magaña, C.; Ubelaker, D. H.: Interpretation of postmortem change in cadavers in Spain. Journal of Forensic Sciences. 2004; 49 (5): I-6.

- Ramos de Pablo, R.; Saloña, M.; Sarasola, E.; Cardoso, S.; Martínez de Pancorbo, M.: Molecular identification of Stearibia nigriceps. An example of the usefulness of Cytochrome $b$ gene for the identification of entomofauna species. International Congress Series. 2006; 1288: 864-866.

- Ribas Ozonas, B.; García Arribas, 0.; Pérez Calvo, M.: Alternative Bestimmung der Wirkung verschiedener Verbindungen mit Dipterae larvae. Mengen und Spurenelemente. 1998; I: I57-164.

- Ribas Ozonas, B.; García Arribas, 0.; Pérez Calvo, M.: Evaluación de toxicidades de residuos metálicos de origen industrial mediante un ensayo con larva de díptero. Actas del V Congreso Internacional de Química de la ANQUE. 1999; 2: 357-365.

- Ribas Ozonas, B.; García Arribas, 0.; Pérez Calvo, M.: 
Metallthioneinisoformen mittels KZE und HPLC in Flechte, Forelle, Dipteralarvae, Ratte, Hase und Mensch. Mengen und Spurenelemente. 1999; I: 948-956.

- Ribas Ozonas, B.; García Arribas, 0.; Pérez Calvo, M.; Labrousse, H.: Diptera larvae as an alternative bioassay in toxicology. ATLA: Alternatives to Laboratory Animals. Proceedings of the Third World Congress on Alternatives and Animal Use in the Life Sciences. 1999; Special Issue, (27): 325.

- Ribas Ozonas, B.; García Aribas, 0.; Pérez Calvo, M.; Novelli, E. L. B.; Escribano, J. M.: Effects of magnetic fields $50 \mathrm{hz} 2.7 \mathrm{mt}$ on physiological parameters during ontogeny of diptera $P$. argyrostoma. Proceedings of the First European Forensic Entomology Seminar. 2002. II7-I26.

- Ríos, T.: Los insectos y la putrefacción de los cadáveres (I-II). La Clínica Moderna, Revista de Medicina y Cirugía. 1902; I (2): 74-80.

- Ríos, T.: Los insectos y la putrefacción de los cadáveres (III-IV). La Clínica Moderna, Revista de Medicina y Cirugía. 1902; I (4): I7I-180.

- Romera, E.; Arnaldos, M. I.; González-Mora, D.; García M. D.: Los Sarcophagidae (Insecta, Diptera) de un ecosistema cadavérico en el sureste de la Península Ibérica. Anales de Biología. 2003; 25:49-63.

- Romero Palanco, J. I.; Munguía Girón, F. J.: Contribución experimental al conocimiento de la entomología tanatológica en la provincia de Cádiz (Sur de España). Actas de las VII Jornadas Mediterráneas de Medicina Legal. 1986. I3I-I44.

- Saloña, M \& González-Mora, D.: Primera cita de Liosarcophaga aegyptica (Salem,1935) (Diptera, Sarcophagidae) de la Peninsula Ibérica, con descripción de sus fases larvarias II y III, pupario y adultos. Boletín de la S. E. A. 2005; 36: 251-255.

- Saloña Bordas, M.: Primera cita de Sarcophaga (Parasarcophaga) albiceps Meigen, 1826 en el País Vasco (Diptera, Sarcophagidae). Boletín de la Sociedad Entomológica Aragonesa.

\section{BIBLIOGRAFÍA:}

I. Gupta, A.; Setia, P.: Forensic Entomology - Past, Present and Future. Aggrawal's Internet Journal of Forensic Medicine and Toxicology. 2004; 5 (I): 50-53.

2. Amendt, J.; Krettek, R.; Niess, C.; Zehner, R.; Bratzke, H.: Forensic Entomology in Germany. Forensic Science International. 2000; II3: 309-314.

3. Amendt, J.; Krettek, R.; Zehner, R.: Forensic Entomology. Naturwissenschaften. 2004; 91: 51-65.

4. Benecke, M.: A brief history of Forensic Entomology. Forensic Science International. 2001; 120: 2-14.

5. Redi, F. Esperienze intorno alla generazione degl'insetti. All'Insegna della Stella. Florencia, 1668.

6. Orfila, M. J. B.; Lesueur, 0.: Traité des exhumations juridiques, et considérations sur les changements physiques dans l'eau, dans les fosses d'aissance et dans le fumier. Béchet Jeune. París, I83I. 331-333.
2006; 39: 409-410.

- Villalaín Blanco, J. D.: Valoración médico-legal de los coleópteros necrófagos de España. Archivos de la Facultad de Medicina de Madrid. 1976; 29 (2): 89-99.

\section{Aplicación a casos reales}

- Arnaldos M. I.; Romera, E.; Luna, A \& García, M. D.: Aportación a la aplicación práctica de evidencias entomológicas en casos forenses en España (serial on line). Revista brasileira de medicina legal. 2004; 2 (I).

- Arnaldos, M. I.; Sánchez, F.; Álvarez, P. \& García, M. D.: A forensic entomology case from the southeastern Iberian Peninsula. Aggrawal's Internet Journal of Forensic Medicine and Toxicology. 2004; 5(I):22-25.

- Arnaldos M. I.; García, M. D.; Presa, J...; Romera, E. \& Luna, A.: Estimation of posmortem interval in real cases on the basis of experimentally obtained entomological evidence. Forensic Science Internacional. 2005; 149: 57-65.

- González Mora, D.; Peris, S. V.; Sánchez Pérez, J. D.: Un caso de entomología forense. Revista Española de Medicina Legal. 1990; 17 (62-63 / 64-65): 19-21.

- Maestre; Pascual; Piga; Fraile; Soler; Aznar; Petinto; PérezMarín; González-Bernal. Informe médico-legal respecto a un mechón de cabellos, de unos insectos y de unos pequeños trozos de piel procedentes de un cadáver decapitado hallado en la estación del Mediodía de Madrid, en un cajón, procedente de Barcelona. Revista de Medicina Legal. 1954; 9 (100-10I): 227248.

- Magaña, C.: Sarcophaga haemorrhoidalis. Revista Española de Medicina Legal. 1999; 23 (86-87): 84-85.

- Villalaín Blanco, J. D.; Bujan Varela, J.: Estudio de un cuerpo momificado hallado en Colmenar Viejo (Madrid). Revista Española de Medicina Legal. 1981; 26-27: 58-68.

7. Orfila, M. J. B.: Tratado de medicina legal, traducido de la cuarta edición y arreglado a la legislación española por el doctor D. Enrique Ataide. Tomo I. Imprenta de José María Alonso. Madrid, 1847. 444-728. 8. Mata, P.: Vade Mecum de Medicina y Cirugía Legal. Volumen II. Imprenta de Manini y compañía. Madrid, I844. 66-72.

9. Solís Claras, L.: Estudio acerca de la conservación provisional de los cadáveres y piezas anatómicas [Manuscrito]. Tesis doctoral, Universidad Central. Madrid, 1885. 26-28 / II7.

10. López Sañudo, L.: Estudios postmortem verificados en el necroscomio y cementerio de Torrero en Zaragoza [Manuscrito]. Tesis doctoral, Universidad Central. Madrid, 1891. 26-28.

II. Bergeret, M.: Infanticide, momification du cadavre. Découverte du cadavre d'un enfant nouveau-né dans une cheminée oú il s'était momifié. Détermination de l'époque de la naissance par la présence de nimphes et de larves d' insectes dans le cadavre et par I' étude de leurs métamorphoses. Annales d'Hygiène Publique et de Médecine Legale. 1855; 4: 442-452. 
12. Mata, P.: Tratado de Medicina y Cirugía Legal teórica y práctica seguida de un compendio de Toxicología. Tomo II. $4^{\mathrm{a}}$ ed. BaillyBailliere. Madrid, 1866. 924-1012.

13. Graells, M.: Entomología judicial. Revista de los Progresos de las Ciencias Exactas, Físicas y Naturales. Madrid. 1886; 21: 458-47I.

14. Mégnin, J.P.: La faune de cadavres. Aplication de I' entomologie a la médicine légale. Encyclopeide scientifique des Aides-Mémoire, Masson, Paris Gauthier-Villars. París, 1894. 214.

15. Reverte Coma, J. M.: Antropología Forense. Ministerio de Justicia, Secretaría General Técnica, Centro de Publicaciones. Madrid, 1991. 399-453.

16. Lecha-Marzo, A.: Tratado de autopsias y embalsamamientos. Ed. Los Progresos de la Clínica. Madrid, 1917. 79-90.

17. Álvarez Herrera, A.: Tanatología forense. Salvat. Barcelona, 1940. 28-30.

18. Piga, A.: Medicina Legal de Urgencia. La autopsia judicial. Ed. Mercurio. Madrid, 1928. 169-179.

19. Domínguez Martínez, J.; Gómez Fernández, L.: Observaciones en torno a la entomología tanatológica. Aportación experimental al estudio de la fauna cadavérica. Revista Ibérica de Parasitología. 1957; 17 (I-2): 3-30.

20. Villalaín Blanco, J. D.: Valoración médico-legal de los coleópteros necrófagos de España. Archivos de la Facultad de Medicina de Madrid. 1976; 29 (2): 89-99.

2I. Sánchez Piñero, F.: Analysis of spatial and seasonal variability of carrion beetle (Coleoptera) assemblages in two arid zones of Spain. Environmental Entomology. 1997; 26 (4): 805-814.

22. Castillo, M.: Estudio de la entomofauna asociada a cadáveres en el Alto Aragón (España). Monografías de la Sociedad Entomológica Aragonesa. Zaragoza, 2002; 6: 1-93.

23. Ríos, T.: Los insectos y la putrefacción de los cadáveres (I-II). La Clínica Moderna, Revista de Medicina y Cirugía. 1902; I (2): 74-80.
24. Ríos, T.: Los insectos y la putrefacción de los cadáveres (III-IV). La Clínica Moderna, Revista de Medicina y Cirugía. 1902; I (4): I7I-I80.

25. Báguena, L.: Algunas notas sobre entomología médico-legal. Graellsia. 1952; 10: 67-101.

26. Arnaldos M. I.; Romera, E.; Presa, J...; Luna, A \& García, M. D. Studies on seasonal arthropod succession on carrion in southeastern Iberian Peninsula. International Journal of Legal Medicine. 2004; II8: 197-205.

27. Martínez-Sánchez, A. I.; Rojo, S.; Marcos-García M. A.: Annual and spatial activity of necrophilus flies (Diptera: Calliphoridae, Muscidae) in a mediterranean holm oak ecosystem. Medical and Veterinary Entomology. 2000; 14:56-63.

28. Martínez-Sánchez, A. I.; Rojo, S.; Marcos-García M. A.: Sarcofágidos necrófagos y coprófagos asociados a un agrosistema de dehesa (Diptera, Sarcophagidae). Boletín de la Asociación Española de Entomología. 2000; 24 (3-4): 17I-185.

29. Martínez-Sánchez, A. I.; Rojo, S.; Rognes, K.; Marcos-García, M. A.: Califóridos con interés faunístico en agroecosistemas de dehesa y catálogo de las especies ibéricas de Polleniinae (Diptera: Calliphoridae). Boletín de la Asociación Española de Entomología. 1998; 22 (I-2): I7I-183.

30. Prieto, J. L.; Magaña, C.; Ubelaker, D. H.: Interpretation of postmortem change in cadavers in Spain. Journal of Forensic Sciences. 2004; 49 (5): I-6.

3I. Vincent, C.; Kevan, D. K.; Leclercq, M.; Meek, C. L.: A Bibliography of Forensic Entomology. Journal of Medical Entomology. 1985; 22 (2): 212-219.

32. Goff, M.L. García, M. D.; Arnaldos M. I.; Romera, E. \& Luna,A.: "Entomología cadavérica: Fundamentos y aplicación. Referencia a la entomología española”. En: Tratado de Medicina Legal y Toxicología. $6^{\mathrm{a}}$ ed. Editado por E. Villanueva Cañadas. Masson. Barcelona, 2004. 253-273. 